\title{
Custos da implantação de um protocolo de prevenção de úlceras por pressão em um hospital universitário
}

\section{Implementation costs of a prevention protocol for pressure ulcers in a university hospital}

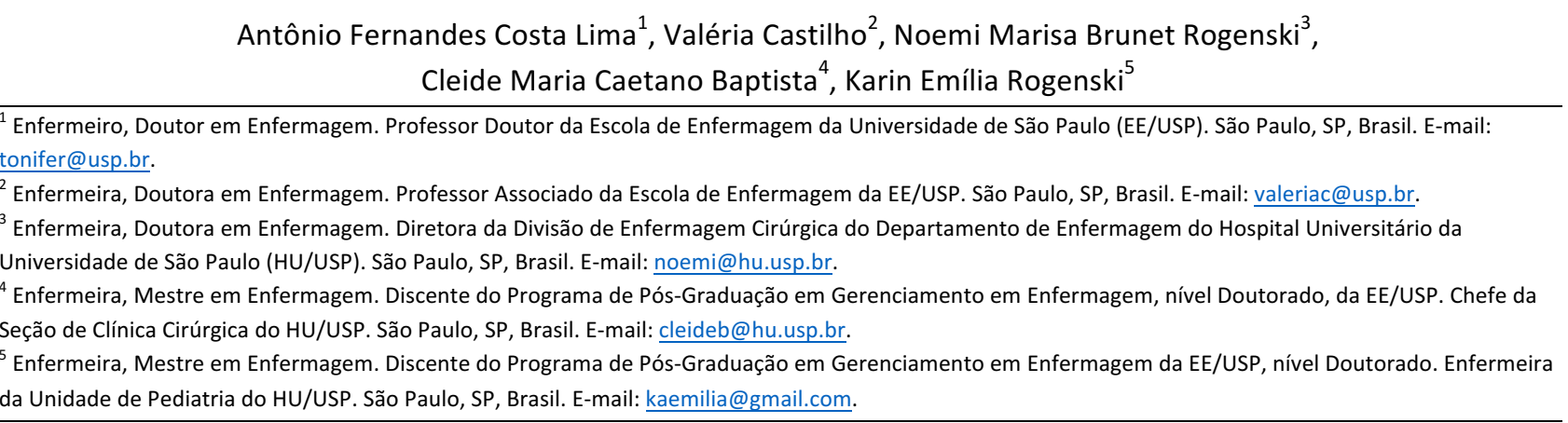

\section{RESUMO}

Pesquisa quantitativa, exploratório-descritiva, do tipo estudo de caso, objetivando calcular os custos diretos da implantação de um protocolo de prevenção de úlceras por pressão (UPs) em um hospital universitário. Foram mapeadas as atividades constituintes das etapas elaboração, implantação e avaliação da implantação do protocolo. O salário hora/profissional foi multiplicado pelo tempo despendido em cada atividade e o custo unitário dos produtos, acessórios e equipamentos multiplicado pela quantidade adquirida para a viabilização do protocolo. A moeda brasileira (R\$) utilizada originalmente para os cálculos foi convertida para a moeda norte-americana pela taxa de US\$ 0.49/R\$. Os custos totalizaram US\$60,857.38 (100\%), sendo US\$ 38,297.64 (62,93\%) relativos à mão de obra direta de enfermeiros, técnicos/auxiliares de enfermagem e secretária, e US\$ 22,559.74 (37,07\%) referentes à aquisição de produtos, acessórios e equipamentos. Espera-se que os resultados obtidos contribuam para subsidiar discussões acerca da imprescindibilidade de investimentos financeiros à efetivação de medidas preventivas de UPs.

Descritores: Úlcera por Pressão; Enfermagem; Custos e Análise de Custo; Controle de Custos.

\section{ABSTRACT}

A quantitative, descriptive, and exploratory case study, aiming to calculate the direct implementation costs of a prevention protocol for pressure ulcers (PUs) in a university hospital. We mapped the activities constituting the creation steps, the implementation, and assessment of the protocol implementation. The hourly salary/professional was multiplied by the time spent in each activity and the costs of each product, accessory and equipment multiplied by the quantity acquired for the protocol feasibility. The Brazilian currency ( $R \$$ ) originally used in the calculations was converted to the North-American currency using the rate US\$ 0.49/R\$. The costs totalized US\$ 60,857.38 (100\%), being US\$ $38,297.64(62,93 \%)$ referred to the direct labor of nurses, technicians/nursing assistants and secretary, and US\$ $22,559.74(37,07 \%)$ referring to the acquisition of products, accessories and equipment. We expect the obtained results to contribute by subsidizing discussions regarding the indispensability of financial investments to implement preventive measures for PUs.

Descriptors: Pressure Ulcer; Nursing; Costs and Cost Analysis; Cost Control. 


\section{INTRODUÇÃO}

O desenvolvimento de úlceras por pressão (UPs) impõe sobrecarga física, emocional e social para o paciente e família. Concorre para a piora da qualidade de vida e aumento dos custos para os serviços de saúde à medida que resulta em maior tempo de hospitalização e aumenta o índice de morbidade e mortalidade ${ }^{(1)}$.

Estudos evidenciam que as UPs representam enorme encargo financeiro para os serviços de saúde e contribuem para a elevação total de custos com cuidados ao paciente, pois quanto mais avançadas as categorias das lesões, mais elevados serão os custos com o tratamento $^{(2-3)}$.

Assim, é muito mais vantajoso prevenir a ocorrência de UPs do que tratá-las. A redução da incidência de UPs diminuiria os custos com curativos e antibióticoterapia; as equipes de enfermagem estariam comprometidas com outros cuidados que não os relacionados às lesões e haveria melhora da qualidade de vida do paciente ao evitar-se o desgaste devido ao aumento do tempo de hospitalização(4).

Além disso, a adoção de um protocolo de prevenção de UPs, ao favorecer atendimento rápido e efetivo, padronizar condutas e diminuir custos, auxiliaria também a minimizar os custos intangíveis correspondentes ao impacto da situação da doença na qualidade de vida da pessoa, às perdas associadas, à presença de dor e de sofrimento que também se estende aos seus entes significativos $^{(5)}$.

Portanto, os profissionais de saúde ao tomarem conhecimento sobre os fatores de risco para a ocorrência de UPs precisam assumir em sua prática clínica a avaliação diária dos pacientes visando programar medidas preventivas eficazes, efetivas, eficientes e, consequentemente, evitar a ocorrência de suas penosas complicações.

Após a avaliação global do paciente ingressante no sistema de saúde, deve ser realizada uma avaliação específica da presença ou risco de desenvolvimento de UPs, incluindo a investigação da etiologia e uma avaliação detalhada da ferida. A evidência sugere que a existência de uma úlcera por pressão (UP) de categoria I é um fator de risco significante para o desenvolvimento de uma UP mais grave ${ }^{(6)}$.

A etiologia das UPs inclui fatores intrínsecos e extrínsecos ao indivíduo como exemplos idade, comorbidades, condições de mobilidade, estado nutricional e nível de consciência. Apesar de sua ocorrência extrapolar os cuidados dos profissionais de enfermagem esses têm se responsabilizado pela implementação de medidas preventivas e sistematizadas de cuidado adotando protocolos baseados em diretrizes internacionais ${ }^{(1)}$.

Nas últimas décadas a preocupação com a estruturação e implantação de protocolos de prevenção de UPs, nos hospitais brasileiros, tem sido enfatizada em decorrência dos programas de melhoria da qualidade que destacam a incidência de UP como um indicador da qualidade da assistência de enfermagem ${ }^{(7)}$.

$\mathrm{O}$ cuidado de enfermagem aos pacientes portadores de UPs além de envolver o conhecimento das alterações psicológicas e emocionais, complicações decorrentes da infecção e internação prolongada, deve contemplar, também, o conhecimento dos aspectos políticos e os custos financeiros decorrentes destas lesões ${ }^{(8)}$.

Então, para viabilizar e incrementar medidas preventivas, fundamentadas nas melhores práticas baseadas em evidências, em diferentes realidades assistenciais, o enfermeiro precisa conhecer os custos referentes à sua implantação para apoiar suas decisões em relação a eficiência alocativa dos recursos humanos, materiais, físicos e financeiros disponíveis. Nessa direção, a fim de contribuir para o aprofundamento do conhecimento sobre esta temática realizou-se o presente estudo objetivando calcular os custos diretos relativos à implantação de um protocolo de prevenção de úlceras por pressão em um hospital universitário. 


\section{MÉTODO}

Esta pesquisa quantitativa, exploratório-descritiva, do tipo estudo de caso único ${ }^{(9)}$, foi realizada no Hospital Universitário da Universidade de São Paulo (HU-USP) após aprovação da Comissão de Ensino e Pesquisa e do Comitê de Ética em Pesquisa do Hospital (Registro no: 881/09 / SISNEP-CAAE: 0002.0.198.196-09590/05).

O estudo de caso foi utilizado por ser um método útil para responder questões do tipo "como" e "por quê", possibilitando compreender um fenômeno da vida real em profundidade, considerando as condições contextuais, ao adotar múltiplas fontes de evidência ${ }^{(9)}$.

O HU-USP destina-se ao ensino e pesquisa, oferecendo assistência multidisciplinar integral, de média complexidade, com base no perfil epidemiológico da população da área geográfica do Distrito do Butantã do Sistema Único de Saúde (SUS) e aos servidores e estudantes da Universidade. Na época de realização do estudo possuía 247 leitos e um Ambulatório para o atendimento de pacientes nas áreas clínico-cirúrgicas, pediátrica e gineco-obstétrica. Os recursos financeiros do hospital são provenientes da dotação orçamentária da USP e dos serviços prestados ao SUS ${ }^{(10)}$.

O Departamento de Enfermagem (DE) do HU-USP tem como finalidade coordenar, supervisionar e controlar as atividades assistenciais, de ensino e de pesquisa desenvolvidas na área de enfermagem. As enfermeiras integrantes do Grupo de Estomaterapia (GE) do DE acreditam que os pacientes com evidente risco de desenvolvimento de UPs devem ser identificados precocemente $\mathrm{e}$ as medidas preventivas adotadas prontamente.

Cientes de que o tratamento das UPs é oneroso para o paciente e família, para a Instituição e para a sociedade, implantaram em julho de 2005, um protocolo(1) almejando a sua prevenção nas Unidades de Clínica Médica (CM), Clínica Cirúrgica (CCir) e Unidade de Terapia Intensiva Adulto (UTIA) por apresentarem maior incidência de UPs. Para tanto, fundamentaram-se em diretrizes propostas pelo European Pressure Ulcer
Advisory Panel (EPUAP) and National Pressure Ulcer Advisory Panel (NPUAP) e adequadas à realidade Institucional.

Nesse período a $\mathrm{CM}$ possuía 41 leitos para o atendimento de pacientes provenientes das Unidades de Pronto Socorro Adulto (PSA), Ambulatório (Amb.), UTIA e demais Unidades do HU-USP sendo, a maioria, idosos e portadores de doenças crônico-degenerativas. A CCir dispunha de 44 leitos para atendimento de pacientes, no período pré e pós-operatório, que necessitavam de cirurgia geral ou ortopédica. Na Unidade eram admitidos pacientes oriundos do PSA, geralmente para a realização de cirurgias de urgência/emergência, do Amb. para cirurgias eletivas e pacientes transferidos de outras Unidades do Hospital. A UTIA era composta por 20 leitos, sendo 12 destinados aos Cuidados Intensivos e oito leitos aos Cuidados Semi-intensivos. Atendia a pacientes portadores de doenças crônicas agudizadas, também idosos em sua maioria, provenientes das diversas Unidades do HU-USP bem como de outras instituições hospitalares.

Os enfermeiros das Unidades campo de estudo conduzem a assistência por meio do Processo de Enfermagem e aplicam a Escala de Braden a todos os pacientes visando à prevenção da ocorrência de UPs. Pacientes com escore menor ou igual a 11 são considerados de alto risco $(90 \%$ a $100 \%$ de possibilidade de desenvolver UPs); pacientes com escore 12 a 14 de risco moderado $(65 \%$ a $90 \%$ de possibilidade de desenvolver UPs em categoria I ou II) e pacientes com escore 15 e 16 de risco leve (50 a 60\% de possibilidade de desenvolver UPs na categoria I). Caso o escore da Escala de Braden for menor ou igual a 16 , os profissionais de enfermagem devem adotar todas as ações preventivas do protocolo $^{(1)}$.

A coleta de dados foi realizada no período de março a junho de 2012.

Foram identificadas quatro etapas constituintes do processo de implantação do protocolo de prevenção de UPs: elaboração do protocolo, viabilização do protocolo, 
aquisição de produtos, acessórios e equipamentos e avaliação do processo. As atividades relativas a cada etapa foram mapeadas a partir dos registros provenientes de Atas de reuniões do GE, documentações disponíveis nos arquivos do Serviço de Apoio Educacional do DE e complementadas por informações obtidas junto a Diretoria do DE, assegurando a diversidade de fontes à adequada condução de um estudo de caso único ${ }^{(9)}$. Em seguida foi levantado o tempo consumido, em cada atividade, por Enfermeiros (Diretor de Divisão, Chefe e Assistencial), Técnicos/Auxiliares de Enfermagem e Secretária do DE.

A moeda brasileira ( $R \$)$, utilizada originalmente para os cálculos, foi convertida para a moeda norte-americana (US\$) pela taxa de US\$ 0.49/R\$, com base na cotação de 29/06/2012, fornecida pelo Banco Central do Brasil.

O cálculo da mão de obra direta (MOD) fundamentou-se no custo direto, ou seja, no dispêndio monetário que se aplica na produção de um produto ou de um serviço em que há possibilidade de identificação com o produto ou departamento ${ }^{(11)}$. Os custos diretos nas unidades hospitalares compõem-se basicamente de mão de obra, insumos e equipamentos utilizados diretamente no processo assistencial ${ }^{(12)}$.

A MOD refere-se ao pessoal que trabalha diretamente sobre um produto ou serviço prestado, desde que seja possível mensurar o tempo despendido e a identificação de quem executou o trabalho. Compõe-se dos salários, encargos sociais, provisões para férias e $13^{\circ}$ salário $^{(11)}$.

Como ocorreram reajustes salariais na USP, após a implantação do protocolo de UPs em 2005, foram utilizados os valores referentes ao ano de 2012 visando à atualização dos dados. Assim, os salários médios, fornecidos pelo Diretor Financeiro do Hospital de acordo com o quadro de profissionais do DE, CM, CCir e UTIA, corresponderam a: Enfermeiro Diretor de Divisão - US\$ 8,590.98; Enfermeiro Chefe - US\$ 7,703.97; Enfermeiro Assistencial - US\$ 5,607.98; Técnicos/Auxiliares de Enfermagem - US\$ 3,693.39 e Secretária - US\$ 3,963.82.
No HU-USP a carga de trabalho dos profissionais de enfermagem é de 36 horas semanais. Logo, o custo da MOD/hora obtida foi: Enfermeiro Diretor de Divisão - US\$ 59.65; Enfermeiro Chefe - US\$ 53,54; Enfermeiro Assistencial - US\$ 38.95; Técnicos/Auxiliares de enfermagem - US\$ 25.65. A carga de trabalho da Secretária corresponde a 40 horas semanais, sendo o custo da MOD/hora de US\$ 24.79.

A especificação da quantidade total de produtos, acessórios e equipamentos a serem adquiridos para viabilização da implantação do protocolo foi inicialmente estimada tendo como referência a média mensal de 60 pacientes com risco de desenvolvimento de UPs, conforme escore obtido por meio da aplicação da Escala de Braden, internados nas Unidades de CM, CCir e UTIA e, desde 2005, estes insumos foram ajustados e incorporados às cotas de materiais destas Unidades. As informações sobre os valores pagos em 2012, para manutenção das cotas destes insumos, foram fornecidas pela Seção de Material, Almoxarifado e Patrimônio do Hospital e complementadas pela Enfermeira Gerente de Materiais do DE.

O salário hora/profissional foi multiplicado pelo tempo despendido em cada atividade e o custo unitário dos produtos, acessórios e equipamentos multiplicado pela quantidade adquirida para a implantação do protocolo.

\section{RESULTADOS}

Na Tabela 1 evidencia-se que o custo com a MOD envolvida na etapa elaboração do protocolo correspondeu a US\$ 5,240.72, sendo o desenvolvimento do protocolo (estabelecimento da finalidade, objetivos, definição de UP e das intervenções de enfermagem) a atividade que mais consumiu (US\$ 3,907.07) nesta primeira etapa. O Enfermeiro Diretor de Divisão foi o recurso que apresentou maior custo com MOD (US\$ 1,998.43), pois participou de todas as atividades por ser coordenador do GE e responsável pela implantação do 
protocolo nas unidades de CM, CCir e UTIA, perfazendo um total de 33 horas e 30 minutos.

O custo da MOD referente a etapa implantação do protocolo no HU-USP correspondeu a US\$ 29,912.00. Conforme mostra a Tabela 2 a participação de profissionais de enfermagem nos programas de capacitação foi a atividade que mais consumiu recurso (US\$ 19,315.10/92 horas de treinamento) e a categoria Técnico/Auxiliar de Enfermagem apresentou maior custo (US\$ 13,746.72).

Tabela 1: Custo da MOD envolvida na etapa elaboração do protocolo de prevenção de UPs. São Paulo, SP, Brasil, 2012.

\begin{tabular}{cccccc}
\hline Atividades & EDD (US\$) & EC (US\$) & EA (US\$) & S (US\$) & Total (US\$) \\
\hline Desenvolvimento do protocolo & $1,789.64$ & $1,338.62$ & 778.81 & - & $3,907.07$ \\
Confecção do protocolo & 89.48 & - & - & 24.78 & 114.26 \\
Aprovação do protocolo & 119.31 & 321.27 & 778.81 & - & $1,219.39$ \\
\hline TOTAL & $\mathbf{1 , 9 9 8 . 4 3}$ & $\mathbf{1 , 6 5 9 . 8 9}$ & $\mathbf{1 , 5 5 7 . 6 2}$ & $\mathbf{2 4 . 7 8}$ & $\mathbf{5 , 2 4 0 . 7 2}$ \\
\hline
\end{tabular}

EDD - Enfermeiro Diretor de Divisão; EC - Enfermeiro Chefe; EA - Enfermeiro Assistencial; S - Secretária.

Tabela 2: Custo da MOD para viabilizar a etapa implantação do protocolo de prevenção de UPs. São Paulo, SP, Brasil, 2012.

\begin{tabular}{|c|c|c|c|c|c|}
\hline Atividades & EDD (US\$) & EC (US\$) & EA (US\$) & S (US\$) & Total (US\$) \\
\hline $\begin{array}{c}\text { Pesquisa e solicitação de compra de produtos, acessórios e } \\
\text { equipamentos }\end{array}$ & 894.51 & 803.17 & 389.40 & - & $2,087.08$ \\
\hline Desenho de acessórios para posicionamento dos pacientes & 715.86 & 642.54 & 389.40 & - & $1,747.80$ \\
\hline $\begin{array}{c}\text { Solicitação de confecção dos acessórios e compra às } \\
\text { empresas especializadas }\end{array}$ & 298.27 & $2,677.56$ & 116.82 & - & $3,092.65$ \\
\hline Coordenação de reunião para implementação do protocolo & 178.96 & - & - & - & 178.96 \\
\hline Participação na reunião para implementação do protocolo & - & $1,606.34$ & $1,168.21$ & - & $2,774.55$ \\
\hline $\begin{array}{l}\text { Desenvolvimento de programa de capacitação para agentes } \\
\text { multiplicadores do protocolo }\end{array}$ & 477.24 & - & - & - & 477.24 \\
\hline $\begin{array}{l}\text { Realização de programas de capacitação dos profissionais de } \\
\text { enfermagem }\end{array}$ & 238.62 & - & - & - & 238.62 \\
\hline Participação nos programas de capacitação & - & 428.26 & $5,140.12$ & $13,746.72$ & $19,315.10$ \\
\hline TOTAL & $2,803.46$ & $6,157.87$ & $7,203.95$ & 13.746 .72 & $29,912.00$ \\
\hline
\end{tabular}

EDD - Enfermeiro Diretor de Divisão; EC - Enfermeiro Chefe; EA - Enfermeiro Assistencial; TE/AE - Técnico/Auxiliar de Enfermagem.

Segundo a Tabela 3 o custo direto da etapa aquisição de produtos, acessórios e equipamentos necessários para a viabilização da implantação do protocolo correspondeu a US\$22,559.74. Dentre estes insumos os itens que mais consumiram recursos foram o colchão pneumático com compressor (US\$ 9,034.80/30 unidades) almofada redutora de pressão (US\$ 6,366.80/110 unidades) e colchão tipo caixa de ovo - densidade 33 (US\$ 4,638.00/30 unidades).

De acordo com a Tabela 4 o custo da MOD envolvida na etapa avaliação do processo de implantação do protocolo foi de US\$3,144.92, sendo o Enfermeiro Assistencial o recurso que apresentou maior custo com MOD (US\$ 2,025.15), totalizando $52 \mathrm{~h}$ com oito enfermeiras concedendo entrevistas, de em média 30 minutos, e 12 participando de um grupo focal com duração de 3 horas e 30 minutos.
A Tabela 5 indica que os custos diretos de implantação totalizaram $\mathrm{R} \$ 123.012,50$. As atividades que mais consumiram recursos relacionaram-se a implantação do protocolo de prevenção de UPs (US\$ 29,912,00) e aquisição de produtos, acessórios e equipamentos (US\$22,559.74). 
Tabela 3: Custo da etapa aquisição de produtos, acessórios e equipamentos necessários à implantação do protocolo de prevenção de UPs. São Paulo, SP, Brasil, 2012.

\begin{tabular}{|c|c|c|c|}
\hline Produtos, acessórios, equipamentos & Valor unitário (US\$) & Quantidade & Total (US\$) \\
\hline Ácidos Graxos Essenciais (AGE) - frasco $200 \mathrm{ml}$ & 2.47 & 152 & 375,44 \\
\hline Protetor Cutâneo Cavilon ${ }^{\circledR}$ - frasco 28 ml & 41.00 & 05 & 205.00 \\
\hline Curativo Hidroativo $10 \mathrm{~cm} \times 10 \mathrm{~cm}$ extra-fino - unidade & 6.93 & 170 & $1,178.10$ \\
\hline Almofada redutora de pressão - unidade & 57.88 & 110 & $6,366.80$ \\
\hline Travesseiro - unidade & 10.88 & 70 & 761.60 \\
\hline Colchão tipo caixa de ovo - densidade 33 -unidade & 61.84 & 75 & $4,638.00$ \\
\hline Colchão pneumático e compressor - unidade & 301.16 & 30 & $9,034.80$ \\
\hline TOTAL & - & - & $22,559.74$ \\
\hline
\end{tabular}

Tabela 4: Custo da MOD relativa a etapa avaliação da implantação do protocolo de prevenção de UPs. São Paulo, SP, Brasil, 2012.

\begin{tabular}{|c|c|c|c|c|}
\hline Atividade & EDD (US\$) & EC (US\$) & EA (US\$) & Total (US\$) \\
\hline $\begin{array}{l}\text { Condução de estratégias (entrevistas e grupo focal) para avaliação da } \\
\text { implantação do protocolo }\end{array}$ & 477.23 & - & - & 477.23 \\
\hline Concessão de entrevistas e participação em grupo focal & - & 642.54 & $2,025.15$ & $2,667.69$ \\
\hline TOTAL & 477.23 & 642.54 & $2,025.15$ & $3,144.92$ \\
\hline
\end{tabular}

EDD - Enfermeiro Diretor de Divisão; EC - Enfermeiro Chefe; EA - Enfermeiro Assistencial.

Tabela 5: Demonstrativo dos custos diretos totais referentes às etapas do processo de implantação do protocolo de prevenção de UPs. São Paulo, SP, Brasil, 2012.

\begin{tabular}{|c|c|c|}
\hline Atividades desenvolvidas / produtos, acessórios e equipamentos adquiridos & Custos diretos (US\$) & $\%$ \\
\hline Elaboração do protocolo & $5,240.72$ & 8,61 \\
\hline Implantação do protocolo & $29,912,00$ & 49,15 \\
\hline Aquisição de produtos, acessórios e equipamentos & $22,559.74$ & 37,07 \\
\hline Avaliação da implantação do protocolo & $3,144.92$ & 5,17 \\
\hline TOTAL & $60,857.38$ & 100,00 \\
\hline
\end{tabular}

\section{DISCUSSÃO}

O aprimoramento da prática da enfermagem tem sido foco de muitas discussões e reflexões no meio profissional, bem como a participação, competência e qualificação dos enfermeiros em gerenciar informações econômico-financeiras com vistas a encontrar caminhos que fortaleçam a função gerencial na apresentação de resultados coerentes as necessidades da clientela atendida $^{(13-14)}$.

A adoção de protocolos assistenciais possibilita melhorar a qualidade da assistência, reduzir as complicações decorrentes da ocorrência de UPs, o tempo de hospitalização, o índice de mortalidade, os custos terapêuticos, a carga de trabalho da equipe de saúde, além de representar um grande avanço na redução do sofrimento físico e emocional do paciente e seus familiares $^{(15-17)}$.

Os resultados do presente estudo evidenciaram o expressivo investimento do DE do HU-USP em MOD (US\$
29,912.00) para viabilização da implantação do protocolo de prevenção de UPs, sendo a realização de programas de capacitação dos profissionais de enfermagem a atividade que mais consumiu recurso (US\$ 19,315.10). Foram desenvolvidos 23 programas teórico-práticos, com duração de quatro horas cada, dos quais participaram 33 (71\%) Enfermeiros e 134 (78,4\%) Técnicos/Auxiliares de Enfermagem atuantes nas Unidades de CM, CCir e UTIA. A categoria Técnico/Auxiliar de Enfermagem foi o recurso que apresentou o maior custo (US\$13,746.72) com MOD em decorrência do elevado número de participantes.

Estudos $^{(4,6,15-17)}$ têm evidenciado a importância da realização dos treinamentos direcionados à equipe de enfermagem, utilizando um protocolo de medidas preventivas, para que sejam identificados os indivíduos em risco e os fatores críticos de desenvolvimento de UPs. À vista disso, é essencial favorecer a qualificação da equipe de enfermagem a fim de desenvolver competências para avaliar o risco de o paciente 
desenvolver UPs e planejar as melhores ações preventivas $^{(6)}$.

Nessa direção, a educação continuada é fundamental para a tomada de consciência da necessidade da formação profissional sistemática, programada de modo a atingir todos os níveis, oferecendo a todos a oportunidade de crescimento profissional e pessoal ${ }^{(18-19)}$.

Contudo, enfatiza-se que na busca da melhoria contínua dos cuidados de enfermagem é imprescindível, também, a adequação quantitativa dos profissionais e a oferta de condições de trabalho que possibilitem o exercício apropriado de suas funções e o atendimento das demandas e expectativas do paciente/cliente ${ }^{(20)}$, neste caso a prevenção da ocorrência de UPs.

Além disso, não se pode imaginar que seja dever apenas das equipes de enfermagem a solução dessa complexa problemática. Há argumentação a favor da formação de equipes multiprofissionais que busquem soluções baseadas em trabalhos científicos, trazendo suas descobertas para a realidade e para a prática diária de cada instituição ${ }^{(4)}$.

Como citado anteriormente, a relevância da adoção de ações preventivas de UPs é irrefutável, especialmente na tentativa de evitar os custos intangíveis que, ao se referirem ao sofrimento físico e/ou psíquico, são os mais difíceis de serem medidos ou valorados, pois dependem da percepção que o paciente tem sobre seus problemas de saúde e as suas consequências sociais ${ }^{(5)}$.

Destarte, está posta a obrigatoriedade da adoção de recursos tecnológicos a fim de se evitar a ocorrências de UPs e suas onerosas complicações. O custo da aquisição de produtos, acessórios e equipamentos para implementação do protocolo nas Unidades de CM, CCir e UTIA correspondeu a US\$22,259.74. Dentre os produtos, acessórios e equipamentos os itens que mais consumiram recursos foram o colchão pneumático com compressor (US\$ 9,034.80); almofada redutora de pressão (US\$ 6,366.80) e colchão tipo caixa de ovo - densidade 33 (US\$ $4,638.00)$.
No caso de pacientes com UPs de categoria I ou II é recomendado, além da supervisão constante da pele, a fim de identificar alterações, e do reposicionamento no leito, o uso de colchões de alta especificação ou almofadas com capacidade de reduzir a pressão como medidas estratégicas para o controle da evolução das lesões e prevenção da ocorrência de novas UPs ${ }^{(21)}$.

Frente ao grande desafio do processo de gestão de custos na área da saúde pública brasileira, destaca-se a necessidade de melhoria da gestão de custos dos hospitais universitários que, por serem referência em tecnologia, precisam oferecer atividades de alta tecnologia, com eficiência no uso dos recursos. Assim, devem, minimamente, conhecer os custos dos procedimentos que realizam ${ }^{(13-14)}$.

A mensuração e o controle dos custos hospitalares são relevantes para identificação e elaboração de estratégias eficazes de contenção de custos, conhecimento da rentabilidade dos diversos procedimentos e serviços, comparação e determinação da correta alocação dos recursos entre os seus diversos setores $^{(22)}$. Nesse sentido, o planejamento da aquisição dos insumos destinados à implantação do protocolo de prevenção de UPs nas unidades de internação do HU-USP foi cuidadosamente conduzido por enfermeiras, com ampla experiência clínica e gerencial, que levaram em conta os avanços tecnológicos, as necessidades dos pacientes atendidos e a viabilidade econômica para atendê-las.

Então, salienta-se a pertinência da projeção do uso racional de materiais médico-hospitalares à administração dos recursos das instituições públicas de saúde no Brasil, tendo em vista que as despesas nesta área são crescentes, enquanto os recursos disponibilizados não aumentam na mesma proporção ${ }^{(4)}$.

Portanto, os profissionais de saúde precisam valorizar os aspectos financeiros da assistência entendendo que gerenciá-los pressupõe o incremento dos recursos para garantir o acesso e a equidade aos usuários com a manutenção da qualidade do atendimento. No entanto, 
eles representam mais uma ferramenta a ser utilizada nos processos decisórios e não devem sobrepor-se aos aspéctos técnicos, humanos, éticos e sociais ${ }^{(12) .}$

\section{CONCLUSÃO}

Os enfermeiros, na condição de gerentes das unidades assistenciais e coordenadores do plano de cuidados dos pacientes, precisam conhecer e se apropriar

\section{REFERÊNCIAS}

1. Rogenski NMB, Kurcgant $P$. The incidence of pressure ulcers after the implementation of a prevention protocol. Rev Lat Am Enfermagem [Internet]. 2012 [acesso em: $31 \mathrm{dez}$.

2015];20(2):333-9. Disponível em:

http://dx.doi.org/10.1590/S0104-11692012000200016.

2. Lyder $\mathrm{CH}$. The benefits of a multi-disciplinary approach to the prevention and treatment of pressure ulcers. Infection Control Today [Internet]. 10 August 2011 [acesso em: $31 \mathrm{dez}$. 2015]. Disponível em:

http://www.infectioncontroltoday.com/news/2011/08/thebenefits-of-a-multi-disciplinary-approach-to-the-preventionand-treatment-of-pressure-ulcers.aspx.

3. Sullivan N, Schoelles KM. Preventing in-facility pressure ulcers as a patient safety strategy. Ann Intern Med [Internet]. 2013 [acesso em: 31 dez. 2015];158(5 Pt 2):410-6. Disponível em: http://dx.doi.org/10.7326/0003-4819-158-5-20130305100008.

4. Lima ACB, Guerra DM. Avaliação do custo do tratamento de úlceras por pressão em pacientes hospitalizados usando curativos industrializados. Ciênc. saúde coletiva. 2011 Jan [cited 2015 Apr 30] ; 16(1): 267-77. Available from: http://dx.doi.org/10.1590/S1413-81232011000100029.

5. Pereira SM, Soares HM. Úlceras por pressão: percepção dos familiares acerca do impacto emocional e custos intangíveis. Referência 2012; Série 3(7): 139-48.

6. Moore Z, Cowman S, Posnett J. An economic analysis of repositioning for the prevention of pressure ulcers. J Clin Nurs. 2013;22(15-16):2354-60.

7. Rolim JA, Vasconcelos JMB, Caliri MHL, Santos IBC. Prevenção e tratamento de úlceras por pressão no cotidiano de enfermeiros intensivistas. Rev Rene. 2013;14(1):148-57.

8. Andrade MM. Avaliação econômica da prevenção de úlceras de pressão em unidade de cuidados intensivos [dissertação]. Vila Real:Universidade de Trás os Montes e Alto Douro;2010. Disponível em: http://hdl.handle.net/10348/2173

9. Yin RK. Estudo de caso: Planejamento e Método. Trad. de Ana Thorell. 5a ed. Porto Alegre: Bookman, 2015.

10. Lima AFC. Treinamento e Qualificação de Profissionais. In: Padilha KG, Vattimo MFF, Silva SC, Kimura M, organizadores. Enfermagem em UTI: cuidando do paciente crítico. São Paulo: Manole; 2010. p.1269-88. de informações financeiras relacionadas à adoção de novas tecnologias e de novas propostas de trabalho.

Diante da ausência de estudos sobre custos para implantação de protocolos de prevenção de UPs, tanto no cenário nacional como internacional, esta pesquisa contribui para o desenvolvimento de um método com potencial de utilização, inclusive para outros protocolos, em diferentes cenários de assistência à saúde.

11. Martins E. Contabilidade de custos. 10å ed. São Paulo: Atlas, 2010.

12. Castilho V, Fugulin FMT, Rapone RR. Gerenciamiento de costos en los servicios de enfermería. In: Kurcgant $P$, Tronchin DMR... [et al.]; traducción Florita Portella Ribas Martins. (Org.). Gerenciamiento en Enfermería. 2a ed. Rio de Janeiro: Guanabara Koogan, 2012, p. 171-82.

13. Reis LR, Campos JLG, Schnaider TB, Juliano Y, Veiga DF, Ferreira LM. Qualidade em um serviço público de cirurgia plástica: visão do paciente. Rev Med Res. 2011;13(4):237-44. 14. Rocha LR, Veiga DF, e Oliveira PR, Song EH, Ferreira LM. Health Service Quality Scale: Brazilian Portuguese translation, reliability and validity. BMC Health Serv Res. 2013;13:24. 15. Furman GF, Rocha AFD, Guariente MHDDM, Barros SKSDA, Morooka M, Mouro DL. Úlceras por pressão: incidência e associação de fatores de risco em pacientes de um hospital universitário. Rev. enferm. UFPE [Internet].

2010 [cited 2015 Apr 30]; 4(3): 169-77. Available from: http://www.ufpe.br/revistaenfermagem/index.php/revista/log in.

16. Spetz J, Brown DS, Aydin C, Donaldson N. The value of reducing hospital-acquired pressure ulcer prevalence: an illustrative analysis. J Nurs Adm. 2013;43(4):235-41.

17. Lima e Silva EWN, Araújo R A, Oliveira EC, Falcão VTFL. Aplicabilidade do protocolo de prevenção de úlcera de pressão em unidade de terapia intensiva. Rev. bras. ter.

intensiva [Internet]. 2010 June [cited 2015 Apr 30]; 22(2): 175-85. Available from: http://dx.doi.org/10.1590/S0103507X2010000200012.

18. Meneses P, Zerbini T, Abbad G. Avaliação de efeitos de treinamento, desenvolvimento e educação de pessoas. In: Meneses P, Zerbini T, Abbad G. Manual de treinamento organizacional. Porto Alegre: Artmed; 2010.

19. Miyazaki MY, Caliri MHL, Santos CB. Conhecimento dos profissionais de enfermagem sobre prevenção da úlcera por pressão. Rev. Latino-Am. Enferm [Internet]. 2010; 18(6): [10 telas]. Disponível em:

http://www.scielo.br/pdf/rlae/v18n6/pt_22.pdf.

20. Fugulin FMT, Lima AFC, Castilho V, Bochembuzio L, Costa $J A$, Castro $L$ et al . Custo da adequação quantitativa de profissionais de enfermagem em Unidade Neonatal. Rev. esc. enferm. USP [Internet]. 2011 Dez; 45(spe):1582-88. Available from: http://dx.doi.org/10.1590/S0080-62342011000700007. 
21. Chou $R$ et al. Pressure ulcer risk assessment and prevention. Ann Intern med. 2013; 159(1):28-38.

22. Ching HY. Fundamentos de custos e métodos de custeio. In: Manual de custos de instituições de saúde: sistemas tradicionais de custos e sistema de custeio baseado em atividades (ABC). São Paulo: Atlas, 2010. 5-23p.

Recebido: 11/07/2014.

Aceito: 22/04/2015.

Publicado: 31/12/2015. 